\title{
EQUILIBRIUM TIMES FOR THE MULTICANONICAL METHOD
}

\author{
M. L. Guerra and J.D. Muñoz \\ Dpto. de Física, Univ. Nacional de Colombia \\ Bogotá D.C, Colombia \\ E-mail: margue@linuxmail.org,jdmunozc@unal.edu.co \\ Received (received date) \\ Revised (revised date)
}

\begin{abstract}
This work measures the time to equilibrium for the multicanonical method on the 2DIsing system by using a new criterion, proposed here, to find the time to equilibrium, $t_{\mathrm{eq}}$, of any sampling procedure based on a Markov process. Our new procedure gives the same results that the usual one, based on the magnetization, for the canonical Metropolis sampling on a 2D-Ising model at several temperatures. For the multicanonical method we found a power-law relationship with the system size, $L$, of $t_{\mathrm{eq}}=0.27(15) L^{2.80(13)}$ and with the number of energy levels to explore, $k_{\mathrm{E}}$, of $t_{\mathrm{eq}}=0.7(13) k_{\mathrm{E}}^{1.40(11)}$, in perfect agreement with the result just above. In addition, a kind of critical slowing down was observed around the critical energy. Our new procedure is completely general, and can be applied to any sampling method based on Markov processes.
\end{abstract}

Keywords: Multicanonical method, time to equilibrium, Markov processes, flathistogram algorithms

\section{Introduction}

The multicanonical method, first proposed by B. Berg and T. Neuhaus ${ }^{1,2}$ and also known as entropic sampling ${ }^{3}$, is a powerful method to explore the phase space of many physical systems on broad energy ranges. It has been sucessfully employed, not only to the study of both first and second order phase transitions in statistical mechanics ${ }^{4,5,6}$, but also to a plenty of many other areas, from optimization problems in computational sciences ${ }^{7}$ to protein folding ${ }^{8,9,10}$ and even covering rules for quasicrystals ${ }^{11}$ (for a review on the applications of the multicanonical method, see $\left.{ }^{12}\right)$. This method uses a Markov process with an extended Metropolis accepting ratio to sample any state $x$ in the phase space with a probability $P(x)$ proportional to the inverse of the density of sates $g(E)$,

$$
P(x) \propto \frac{1}{g[E(x)]},
$$


with $E(x)$ the energy of state $x$. Then, the Metropolis accepting ratio for a move from $x$ to $x^{\prime}$ becomes

$$
A\left(x^{\prime} \mid x\right)=\min \left\{1, g[E(x)] / g\left[E\left(x^{\prime}\right)\right]\right\} \quad .
$$

By this method, the histogram of samples on the energy axis, $V(E)$, is flat, so long $g(E)$ is the actual density of states. If not, a better approximation $g_{1}(E)$ can be found by dividing $V(E)$ into $P(x)$, i.e.,

$$
g_{1}(x) \propto V(E) g(E) \quad .
$$

The multicanonical method is actually an iterative procedure that, first, proposes some trial function $g(E)$ and, then, improves it by successive applications of the procedure described above.

As every Markov process does, the multicanonical method needs a number $t_{\mathrm{eq}}$ of steps before reaching the desired probability distribution, that is, before starting to take the samples. For some sampling distributions, like the Boltzmann one, it is easy to find narrowed-distributed quantities, like the magnetization, such that $t_{\text {eq }}$ can be measured just by tracking the process until that quantity oscillates around a mean value and inside a $10 \%$ confidence interval, for instance. That is what is called statistical control. The well-established procedure of computing the non-linear correlation function and the non-linear correlation time ${ }^{13}$ is just a sophistication

of this procedure, which gives good results if the quantity evolves to its equilibrium distribution as an exponential decay. For the multicanonical method, in contrast, it is not so easy to imagine a narrow-peaked quantity that can be used for this purpose. The typical procedure to find $t_{\text {eq }}$ is to run two or more instances of the system, and to keep track in all of them of some quantity of interest, i.e. the magnetization, until all instances join together. The time to equilibrium $t_{\mathrm{eq}}$ is taken as the number of steps, measured in Monte Carlo steps per site (MCSS), before this joint. Despite the fact that this second criterion is easy to improve, it deserves the statistical precision of the first one.

This paper introduces a new criterion to measure $t_{\text {eq }}$ and uses it to investigate the time to equilibrium of the multicanonical sampling method. The new criterion is based on the chi-squared $\left(\chi^{2}\right)$ deviation between the desired limit distribution and the actual energy distribution after $t$ steps. First, we introduce this chi-squared procedure, and we test it against the more traditional one, based on the magnetization, for the usual Metropolis sampling with Boltzmann distribution. Second, we use the chi-squared procedure to investigate the time to equilibrium for the multicanonical method on 2D-Ising systems, and how it depends on the system size and the energy. Finally, we summarize our main results and conclusions.

\section{The Procedure}

Consider a sampling method based on a Markov process that takes any state $x$ with some probability $P(x)$, which is function of $E(x)$ alone. Starting from 
some initial distribution $P_{0}(x)$, the Markov process transforms it at every step until it reaches the desired distribution $P(x)$. Let us run $n$ times the Markov process, starting form the same initial distribution $P_{0}(x)$ but running with different seeds of the random number generator. Look at the energy the system has after $t$ steps on each run, and cumulate the histogram $V_{t}(E)$ from these energy values. If $V_{t}(E)$ actually corresponds to the limit distribution $P(x)$, it should approximate the expected probability distribution for $E, P(E)=g(E) P(x)$. The $\chi^{2}$ deviation is a good statistics to verify this point. It is given by

$$
\chi^{2}(t)=\sum_{E} \frac{\left[V_{t}(E)-n P(E)\right]^{2}}{n P(E)}
$$

where the sum extends over all energy values with more than five or ten samples. For large $n$ values, $\chi^{2}$ obeys a chi-square distribution of $r=k_{E}-1$ degrees of freedom, with probability density

$$
P\left(\chi^{2}\right)=\frac{\left(\chi^{2}\right)^{(r-2) / 2} e^{-\chi^{2} / 2}}{\Gamma(r / 2) 2^{r / 2}}
$$

where $k_{E}$ is the number of energy levels in the sum ( ${ }^{19}$. This distribution has population mean $\mu=r$ and standard deviation $\sigma=\sqrt{2 r}$. If we repeat $m$ times the whole study and look at the average $\overline{\chi^{2}}$ of that $m$ values, $\overline{\chi^{2}}$ distributes like a Gaussian, with $\mu=r$ and $\sigma=\sqrt{2 r / m}$. That is, $95,45 \%$ of the values of $\overline{\chi^{2}}$ lies between $r-2 \sqrt{2 r / m}$ and $r-2 \sqrt{2 r / m}$. This is only true if $V_{t}(E)$ corresponds to the limit distribution $P(E)$. That is what is called statistical control ${ }^{14}$.

So, the new procedure to find the time to equilibrium is just to compute $\overline{\chi^{2}}$, as described above, and to look at the time, $t_{e q}$, when the statistical control starts. Figure 1 shows how this criterion works for a Metropolis sampling with limit Boltzmann distribution on a $8 \times 8$-Ising model. The temperature was $T=3.0$, and the system always started from the state of minimal energy. In this example, only $k_{E}=22$ energy levels were taken into account to compute $\chi^{2}(t)$ (Eq. (4)), and the whole study was done $m=10$ times to compute $\overline{\chi^{2}}$, with $n=10^{4}$ runs each. Therefore, statistical control is reached when $\overline{\chi^{2}}$ oscillates around $r=21$, and between $r-2 \sqrt{2 r / m}=16.9$ and $r+\sqrt{2 r / m}=25.1$. We found $t_{e q}=33(3)$.

Figure 2 shows $t_{e q}$ for the same $8 \times 8$-Ising model, when sampled by the Metropolis method with limit Boltzmann distribution at several temperatures. Two criteria were used to measure $t_{e q}$ : the $\chi^{2}$-criterion described above and another one based on the statistical control on the magnetization $M$, as follows: we looked at the magnetization the system had after $t$ steps on each one of the $n$ runs and computed their mean value $\langle M(t)\rangle$. The time to equilibrium was chosen as the time when statistical control started, with $\mu=\langle M\rangle_{T}$ (the average magnetization at temperature $T$ ) and $\sigma=\sqrt{T \chi_{\mathrm{s}}(T) / m}$ (with $\chi_{\mathrm{s}}(T)$ the magnetic susceptibility at temperature $\left.T, \chi_{\mathrm{s}}(T)=1 / k_{\mathrm{B}} T\left[<M^{2}>_{T}-<M>_{T}^{2}\right]\right)$. All other parameters in the simulation were the same as in figure 1 . We can see an excellent agreement between both criteria 


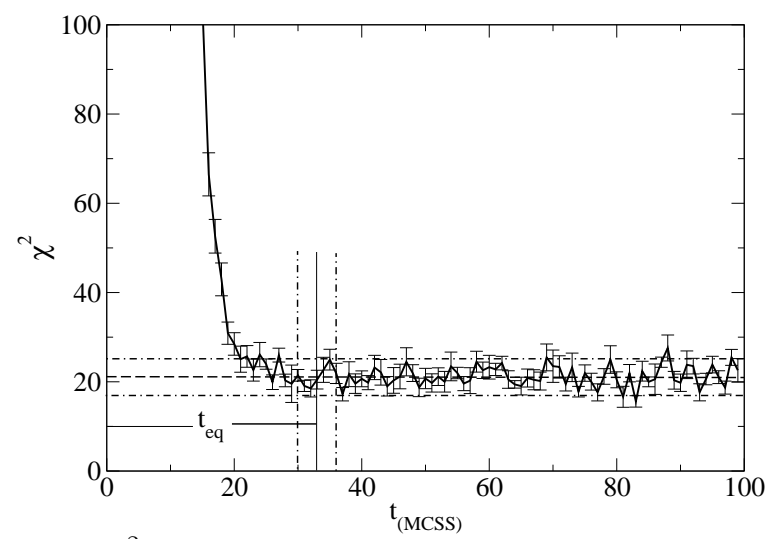

Fig. 1. Example of the $\chi^{2}$-criterion to compute the time to equilibrium $t_{e q}$ for a Metropolis sampling method with limit Boltzmann distribution $(T=3.0)$ on a $8 \times 8$-Ising model.

for all temperatures. That is, our new criterion works well by measuring the time to equilibrium of this Markov process.

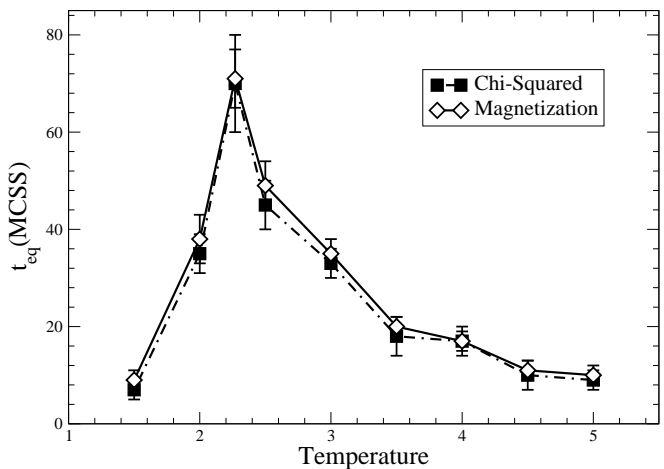

Fig. 2. Time to equilibrium $t_{e q}$ for a Metropolis sampling method with limit Boltzmann distribution at several temperatures on a $8 \times 8$-Ising model, computed both with the new $\chi^{2}$-criterion and by looking at the statistical control on the magnetization. The two criteria agree for all sampled temperatures.

\section{The multicanonical case}

With the new $\chi^{2}$-criterion on hand, we investigate the time to equilibrium for the multicanonical sampling method on two-dimensional Ising models of several sizes. For all simulations the actual density of states $g(E)$, computed by P.D. Beale ${ }^{15}$, was used from start to perform every multicanonical step. In all cases we chose $n=10^{4}$ and $m=10$, as before.

Figure 3 shows time to equilibrium against system size, when the whole energy range of positive temperatures $-2 L^{2} \leq E \leq 0$ is sampled. Through linear regression, 
we obtain a power-law behavior, $t_{\mathrm{eq}}=0.27(15) L^{2.80(13)}{ }^{a}$, with correlation coefficcients $r=0.9988$ and $F=2416.72$. That is, the time to equilibrium increases with system size like $L^{2.80(13)}$.

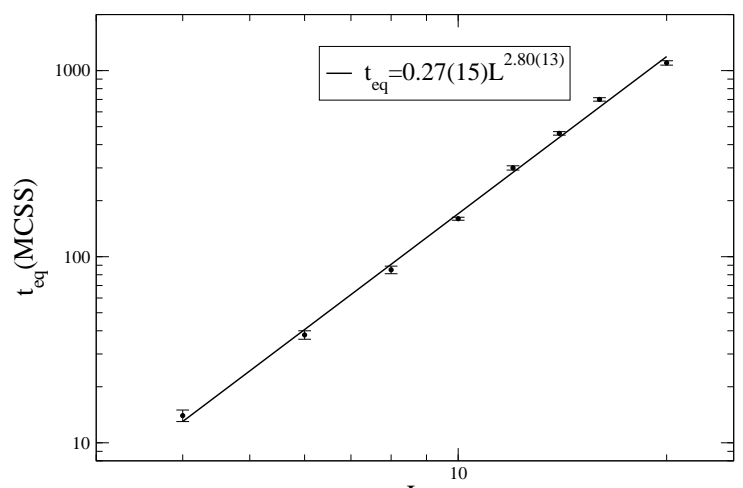

Fig. 3. Dependence of the time to equilibrium $t_{e q}$ on system size $L$ for the multicanonical sampling method on $L \times L$-Ising models. The true density of states $g(E)$ was used from start at every multicanonical step.

Next, we look at how the time to equilibrium changes with the number of energy levels (the number of bins in the histogram) sampled by the multicanonical method. For that purpose a set of multicanonical simulations was performed on Ising models of system sizes $L=6,8,10,12$ and 16. All energy intervals start from $E_{\text {inf }}=-2 L^{2}$ and extend up through $k_{E}$ energy levels. Figure 4 shows $t_{e q}$ against $k_{E}$ for all system sizes. We obtain, again, a power-law behavior, $t_{e q}=0.70(13) k_{E}^{1.40(11)}$, with correlation coefficients $r=0.9783$ and $F=598.19$. Summarizing, $t_{e q}$ grows like $k_{E}^{1.40(11)}$.

Finally, we hold constant the relative length of the energy interval and we investigate how the time to equilibrium changes with the position of this interval along the energy axis. With $E_{\min }=-2 L^{2}$, an energy interval of length $0.16\left|E_{\min }\right|$ was set at different places on the energy axis, actually around approximate energy values of $0.92,0.84,0.75,0.68,0.59,0.52,0.43,0.36,0.25$ and 0.10 times $E_{\text {min }}$. The system reached the energy interval from below by starting at a configuration of minimal energy and by accepting every random flip that increases the energy. This procedure fix the initial distribution $P_{0}(x)$. A whole set of simulations was performed on systems of sizes $L=6,8,10,12$ and 16 , as before. Figure 5 summarizes these

results. We try a finite-size scaling around the critical energy $E_{\mathrm{c}}=0.7540328 E_{\min }$ (the mean energy at the critical temperature $T_{\mathrm{c}} \simeq 2.269185$ for an infinite-size system) by plotting $L^{-2.0} t_{e q}$ against $L^{0.7}\left(E-E_{\mathrm{c}}\right) / E_{\mathrm{c}}$. Although the scaling is not

\footnotetext{
${ }^{a}$ All error bars in this paper are two-sigma.
} 


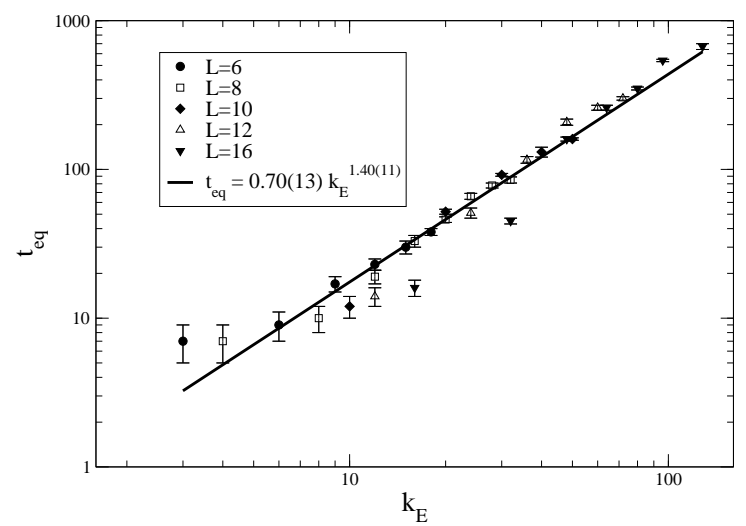

Fig. 4. Time to equilibrium $t_{e q}$ against the number $k_{E}$ of energy levels sampled by the multicanonical method, on two-dimensional Ising models of system sizes $L=6,8,10,12$ and 16 . All energy intervals to 1

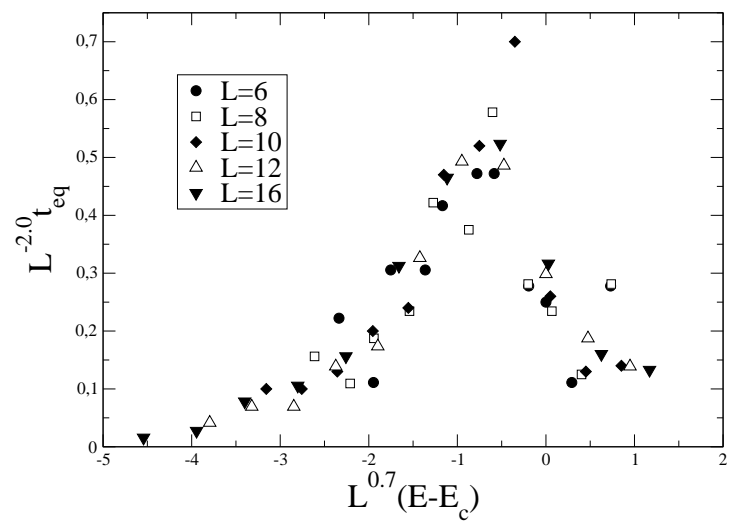

Fig. 5. Time to equilibrium $t_{e q}$ for the multicanonical samplig method on energy intervals of length $0.16\left|E_{\min }\right|$ (with $E_{\min }=-2 L^{2}$ ), placed at different places on the energy axis. The horizontal axis represents the value for the central energy $E$ in the interval. A certain kind of critical slowing down can be observed around a critical energy of $E_{\mathrm{c}}=0.7540328 E_{\min }$. The simulations were performed on two-dimensional Ising systems of sizes $L=6,8,10,12$ and 16 .

a perfect one, we observe a certain kind of critical slowing down, i.e. the time to equilibrium $t_{e q}$ grows if the energy interval lies near the critical energy and diverges with increasing system size.

\section{Conclusions}

As every Markov process does, multicanonical sampling needs some steps before reaching the desired sampling distribution. We have found that this time to equilibrium (measured in MCSS) changes mainly with the number of energy bins, $k_{E}$, to be sampled. Our main result is that this dependence obeys a power law, $t_{e q}=0.70(13) k_{E}^{1.40(11)}$, at least for the two-dimensional Ising model. If one wants to sample the whole energy interval of positive temperature for several system 
sizes (which consists of $k_{E}=L^{2} / 2$ energy bins), this result predicts that $t_{e q}$ will grow with system size, $L$, as $t_{e q}=0.27(5) L^{2.80(22)}$. A direct measurement gave us $t_{\text {eq }}=0.27(15) L^{2.80(13)}$, in perfect agreement with the prediction above.

This result has important consequences. First, it supports the usual procedure with multicanonical samplings of dividing the energy interval of interest in many small intervals and sampling each one separately. This is not just to parallelize the method, it actually reduces the total time to equilibrium, because it grows faster than linear with $k_{E}$. Second, it says us that we have to be much more careful by performing multicanonical samplings on more than one parameter. If we construct an histogram on two parameters (let us say, the energy and the magnetization, for instance) with $k$ bins on each one, the time to equilibrium will grow like $k^{2.80}$, and that can be much faster than expected (so far our results can be extended to this somewhat different - situation).

By dividing the energy range of interest in a fixed number of small intervals and by using a multicanonical sampling on each one, we have found that the equilibration times is larger for energy intervals around the critical energy (the mean energy at critical temperature), and that those times grow with system size like $L^{2.0}$, approximately. Although the finite-size scaling is not perfect, it tells us that we have to be more careful on energy intervals around the critical energy, and that the time to equilibrium should be measured just there, if we want to assign the same number of MCSS equilibration steps for all intervals.

All these results were obtained by using a new criterion to estimate the time to equilibrium of any sampling Markov process. We have shown that this new criterion gives the same equilibration times than the usual one (based on the magnetization) for the canonical Metropolis sampling of a 2D-Ising model at several temperatures. Although general, this new method is especially useful for sampling procedures that construct a flat histogram on the energy axis, like the multicanonical method, the flat-histogram method of J.-S. Wang ${ }^{16}$, the sampling method of F. Wang and D. P. Landau ${ }^{17}$ or the broad-histogram sampling of P.M.C. de Oliveira ${ }^{18}$. We are sure that our criterion will work properly for all those sampling procedures, revealing important issues of its behavior that can encourage its proper use and improve its performance.

\section{References}

1. B. A. Berg, J. Mod. Phys. C3, 1083 (1992).

2. B. A. Berg, Comp. Phys. Comm. 153, 397 (2003).

3. J. Lee, Phys. Rev. Lett. 71, 211 (1993).

4. B. A. Berg and T. Neuhaus, Phys. Rev. Lett. B267, 249 (1991).

5. B. A. Berg and T. Neuhaus, Phys. Rev. Lett. 68 , 9 (1992).

6. B. A. Berg, A. Billoire and W. Janke, Phys. Rev. E65, 045102R (2002).

7. A. R. Lima and M. Argollo de Menezes, Phys. Rev E63, 020106R (2001).

8. Y. Peng, U. H. E. Hansmann and N. A. Alves, J. Chem. Phys. 117, 2374 (2003).

9. H. Arkin and T. Celik, Int. J. of Mod. Phys. C14, 113 (2002). 
10. U. H. E. Hansmann and Y. A. Okamoto, J. Comput. Chem. 14, 1333 (1993).

11. M. Reichert and F. Gahler, cond-mat/0302070, (2003).

12. B. A. Berg, Fields Inst. Commun. 26, 1 (2000).

13. K. Binder and D. W. Heermann, in Monte Carlo Simulation in Statistical Physics:An Introduction (Ed. Heidelberg Springer, 1997), p.150.

14. R. E. Walpole and R. H. Myers, in Probability and statistics for engineersand scientists, (Macmillan Publishing Co., 1991), p.677.

15. P. D. Beale, Phys. Rev. Lett. 76, 78 (1996).

16. J. -S. Wang, Physica A 147, 281 (2000).

17. F. Wang and D. P. Landau, Phys. Rev. Lett. 85, 2050 (2001).

18. P. M. C. de Oliveira, T. J. P. Penna and H. J. Herrmann, Braz.J. of Phys. 26, 677 (1996).

19. R. E. Walpole, in Introduction to statistics, (Macmillan Publishing Co., 1982), p.275. 